\title{
Organizational behaviour analysis and information technology fitness in
} manufacturing: Analysis through modelling and simulation

\author{
A. Lucas Soares, J.J. Pinto Ferreira, J. M. Mendonça \\ INESC/Porto, R. José Falcão, 110 - 4000 Porto, Portugal \\ FEUP/DEEC, Univ. of Porto, R. Bragas, 4099 Porto Codex, Portugal \\ email: asoares, jjpf, jmm@tecno.inescn.pt
}

\begin{abstract}
The analysis and design of integrated manufacturing systems, under a techno-organizational approach, is a complex and time consuming process. It involves people from different knowledge areas that must cooperate in order to build a common and complementary understanding of the enterprise's organization, people and technology. Particularly the SME's, although subjected to the same pressures than their bigger counterparts, have scarcer time, people and technical resources. This paper presents a novel approach to the modelling and simulation of organizational structures and information technology resources in integrated manufacturing systems. The tool resulted from this research work enables the analysis and evaluation of alternative organizational designs and different combinations of group/software task assignment. This approach goes beyond the operational quantitative and logical analysis, enabling the assessment of socio-technical evaluation criteria. Moreover, this tool enhance the cooperation and communication within the multidisciplinary team developing the technoorganizational system: users, technical developers and organization/social specialists.
\end{abstract}

\section{Keywords}

Organization modelling, simulation, techno-organizational development, socio-technical systems.

\section{INTRODUCTION}

Complex restructuring processes are needed to change organization structure and culture in order to accommodate technological innovation in manufacturing. Empirical studies put into focus the influences of information technologies on the organization and people in production systems (Soares, 1994) and the difficulties to put in practice the increasingly accepted "joint development" in all of its dimensions. Anyway, it is generally accepted that the technocentric approach to the integrated manufacturing systems (IMS) development is no longer appropriate (or never has been...), and is given place to anthropocentric approaches.

INESC/Porto (Institute of Systems and Computers Engineering at Porto), besides its R\&D work on advanced information technology systems for manufacturing management, has been undertaking research work in modelling tools supporting IMS development. Two main streamlines emerge from this work: the support to the development, configuration and fine- 
tuning of industrial IT infrastructures and applications and the support to technoorganizational development. The later is the subject of this paper and is achieved through the joint modelling and simulation of organizational structures and the IT shop floor management applications.

\section{INTERDISCIPLINARY TECHNO-ORGANIZATIONAL ANALYSIS}

Advanced information management systems for manufacturing applications have profound implications in the organization where are introduced. The "constructive character" (Bachman, 1994) of these technologies will only be fully exploited if they are understood in a theoretical and conceptual framework that rejects the technocentric view in favour of a vision that contemplates the interaction between the organizational subsystems which technology is part of. The joint development of information technology and organization is only possible if engineering and organizational/social knowledge areas can be applied in a cooperative way. For that, tools have to be developed in order to enhance this cooperation. Technoorganizational analysis is a development phase were a strong cooperation within the development team is needed. Modelling tools are collaboration instruments (Paul, 1994) and must be thoroughly used for an effective analysis.

\subsection{Models and modelling purposes}

Modelling is an increasingly important object of R\&D in IMS analysis and design. To clarify the modelling purpose in the context of techno-organizational analysis and design a simple classification of models is presented below.

\section{Descriptive models}

Descriptive models capture two main properties of a system: structure and behaviour. Structural models represent function and information arrangements within a technical system or an organization. Behaviour models describe the dynamics of those structures. Basic models in this category make use of data flow diagrams, SADT, Entity-Relationship diagrams, objectoriented models, data transition diagrams, and organization charts. Composite models, aggregating or adapting the basic models, are the ones used in modelling frameworks and/or enterprise reference architectures like CIM-OSA, GRAI-GIM and PERA (Williams, 1993).

Descriptive models have a computer science root and have been used mainly for the specification and design of computerised systems. Therefore they are built on a machine metaphor resulting in major drawbacks when it comes to model the real-world of the manufacturing systems (Bansler, 1993, Hoydalsvik, 1993, Kensing, 1993). The first difficulties arose when the increasingly interactivity of those systems called for the systems environment modelling, particularly the human environment. More recently, with the growing awareness of the information technology organizational impact, new difficulties have emerged in the joint modelling of the technical system and the organization, namely in the explicit representation organizational structures and the their social aspects . Besides these drawbacks, there is the problem of how to cope with the modelling complexity due to his deepness (model level of detail) or wideness (model scope). The model construction and the evaluation resulting from model analysis can be cumbersome and time consuming calling for computer based tools that help users in this task.

\section{Evaluation models}

In the analysis and design of IMS, simulation is a frequently used tool to evaluate and compare alternative scenarios for the organization of manufacturing activities and production 
management strategies. Simulation systems rely on computational models that describe the operational aspects of activity networks, enabling their quantitative and logical evaluation. The construction of these models depends on the simulation system. Maximum modelling flexibility is achieved with general purpose simulation languages, but these offer very limited expressiveness for problem communication and understanding. On the contrary, "data-driven" simulation (based on pre-defined models, usually queuing networks), in conjunction with graphical interfaces, provide less flexibility but better communication for the system structure and the concepts under evaluation.

Although simulation is a well established field in the analysis and evaluation of IMS at the physical operations level, there are few contributions to the simulation of organization structures and even fewer when we add the modelling and simulation of software applications supporting manufacturing activities. Organization structure simulation has been addressed from an exclusively operational viewpoint and focusing on evaluation criteria similar to those used in the physical operations level: lead-time, WIP level, system loading and delivery dependability (Zülch, 1993). Also work on shop floor control software and physical operations joint simulation have been reported in (Ferreira, 1994). Here the modelling purpose is the shop-floor software behaviour and their interaction with the physical operations, the goal being to support the technical system development.

\section{Hybrid models}

Modern simulation tools based on object-oriented concepts and equipped with powerful graphical interfaces enable, to certain extent, the integration of descriptive and evaluation modelling. This results in simulation models with structural representation power (functional and informational) in addition to the quantitative and logical description, overcoming the lack of expressiveness of evaluation models. Moreover, object-orientation provides means for the explicit inclusion of "non-functional" information, paving the way to effective organizational modelling.

\section{HYBRID MODELLING FOR TECHNO-ORGANIZATIONAL ANALYSIS}

The work presented here is a contribution to the interdisciplinary development of IMS. It resulted in a simulation based system for the modelling and analysis of organizational and information technology structures in manufacturing. In this section the underlying concepts and theory of the Organization Behaviour simulation system (OBsim) will be presented .

Modelling is a mean, thus it must be clearly stated what its purpose is. OBsim is intended to help the joint development of manufacturing organization and the supporting software applications. For that, evaluation criteria were found in socio-technical systems theory and in industrial psychology. Besides basic quantitative performance indicators, e.g. task/function/process lead time or organizational unit temporal occupation, socio-technical indicators were chosen to be directly or indirectly assessed through OBsim. Examples of these indicators are the completeness of realised work functions, independence of organizational units, task interdependence, boundary regulation, group/software coupling and technical linkage (integration/dependency) (Weik, 1994, Emery, 1978). An evaluation model alone would not fulfil the purpose of a techno-organizational analysis because of the poor communication capability arising from the lack of structural description power. On the other side, the quantitative and logical evaluation of organizational scenarios calls for a simulation system with the corresponding evaluation model. Hybrid modelling is thus necessary to undertake an effective organizational analysis based on socio-technical evaluation criteria. 


\subsection{The Organization Behaviour Simulation system}

\section{Building classes}

The techno-organizational model used in OBsim is outlined in Figure 1. Task and software module are the basic building classes, from a functional viewpoint, being aggregated by function and software system classes. The class organizational unit is linked to tasks and functions through assignment and qualification links. A short description of the functional building classes is given below:

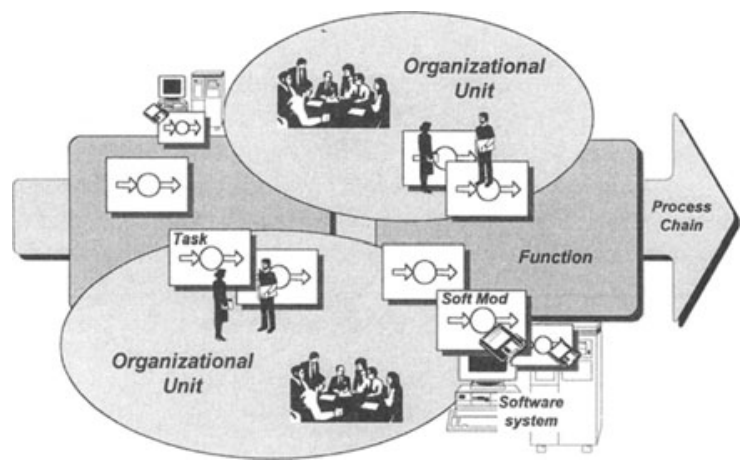

Figure 1 Techno-organizational model structure.

- organizational unit - models elementary units describing the task assignment, qualification structure, capacity, learning process and other relevant information to describe its behaviour; this class can model working groups, manufacturing sections, production cells, production islands, etc.,

- task - describes the basic behaviour of organizational units; it includes, besides the behaviour model, local information for the task fulfilment and evaluation; can be further decomposed in elementary activities,

- function - aggregates closely related tasks, and is the basis for the process chain modelling,

- software module - models elementary functions realised by a software system; the software behaviour description is made from an external (interaction) point of view; can be further decomposed in elementary software activities,

- software system - aggregates software modules that form a self-contained software application; the software model represents essentially the software applications specification, being one of the inputs for the software system construction.

The information network is modelled after two basic building classes:

- operational information - information needed to coordinate and describe the organizational tasks.

- monitoring information - feedback information on the degree of achievement of the task actions.

These classes are further specialized in operational coordination and operational description information flows. The former represents temporal plans that coordinate when organizational units realise their tasks. Examples are production schedules, maintenance plans, etc. The later represents how the organizational units realise their tasks, e.g. product structures, process plans, NC programs, etc. 


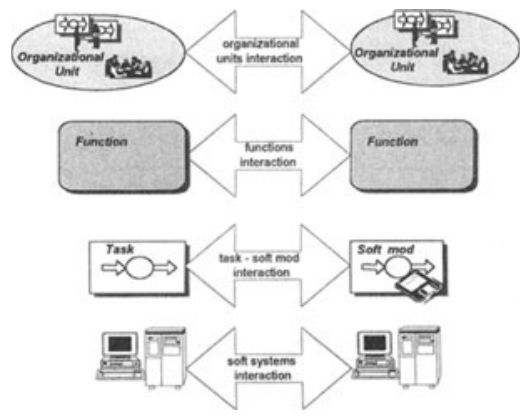

Figure 2 Information flows interaction roles.
During the model construction are given interaction roles to the information flow classes depending on the sender and the receiver of the information. This roles are shown in Figure 2.

The model building classes belong to a class library and are instantiated during the model construction. Furthermore, new classes can be obtained from the existing ones through inheritance mechanisms and added to the library, enabling an effective reuse of previously done analysis.

\section{The modelling and simulation infrastructure}

OBsim is built on a object-oriented simulation tool, SIMPLE++. This tool is primarily intended to simulate manufacturing operations at the physical level, although it incorporates mechanisms to model information control flow. Novel work has been undertaken at INESC/Porto to provide SIMPLE++ with facilities to specify and model shop floor software applications. The resulting integrated modelling environment encompasses both material and information flows, supporting R\&D work in information flow and application software modelling, as well as its full integration with shop floor material flow models. OBsim used the additional facilities described to model and simulate the organizational tasks and software modules.

The behaviour of each function or software system is represented using SDL (Specification and Description Language) which is a design language standardised by ITU-TSS (International Telecommunications Union) for the design of computer based exchange systems. Besides originating from the telecommunications areas and having been used in this field for a long time, SDL is a powerful modelling language and may be applied in many other areas including the design of hybrid hardware \& software systems (Ferreira, 1994). The SDL basic structure and its mapping to the techno-organizational model is shown in Figure 3.

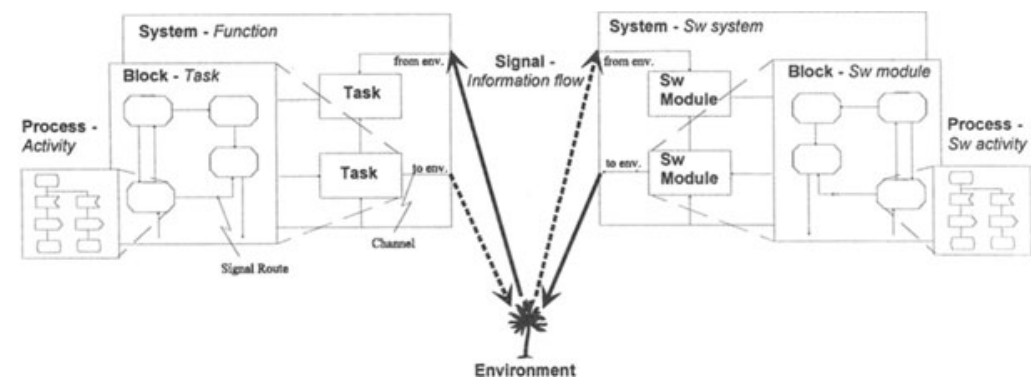

Figure 3 SDL hierarchy and its mapping to the techno-organizational model.

\subsection{Socio-technical analysis and evaluation}

The novel approach in OBsim brought socio-technical analysis criteria into the organizational structures simulation, thereby overcoming the "machine metaphor" which is inherent to quantitative models. Socio-technical analysis criteria can only be fully addressed if descriptive 
and evaluational representations are available. Hybrid models are thus suitable for this analysis. These criteria and the way OBsim can assess them will be briefly described below .

\section{Completeness of realised functions in the organizational unit}

Motivational factors lead to the need of job enrichment in the organizational unit. The processing depth of the realised tasks, i.e. vertical integration of functions, is the way of achieving this goal. In OBsim, vertical integration of functions is structurally assessed by visualising the task assignment together with the defined process chains. The higher the number of tasks belonging to the same process chain is assigned to the organizational unit the higher is the completeness of realised functions.

\section{Independence of the organizational unit}

The degree of dependence of an organizational unit on other units, being either vertical or horizontal, is an important effectiveness factor. The minimisation of this dependence is a requirement of actual organizational paradigms: production islands, autonomous working groups, manufacturing cells. OBsim assesses this criteria in a structural and quantitative way. When an information flow has an organizational units interaction role, it connects two tasks assigned to different organizational units, this representing a dependence link. The link can be further classified as operational or monitoring flow. During a simulation session the intensity of the flows between the organizational units can be recorded. The higher the intensity of flow the lower the organizational unit independence. This is also related with the following criteria.

\section{Function and task interdependence}

The form of cooperation between tasks can be classified after Thompson's categories of interdependence (Thompson, 1967). Three categories are considered:

pooled interdependence which is the lowest form of interdependence meaning that none or very few information flows link the tasks, sequential interdependence that exists when information flows between tasks are uni-directional, reciprocal interdependence is the highest form of interdependence and appears when there are bi-directional information flows between tasks.

As the interdependence increases, it makes greater demands on intra and inter organizational units coordination, communication and decision-making. Again, this criteria is evaluated in OBsim through the monitoring of the intensity and direction of the information flows between tasks and functions.

\section{Organizational unit qualification}

Qualification is modelled through a matrix where a ranking of the tasks that the organizational unit has competence and skills to perform is stored. During simulation the qualification table is used to quantify the efficiency of the organizational unit in realising the assigned tasks. One important feature of OBsim is the possibility to evaluate functional flexibility. This is achieved through a dynamic task assignment that occurs e.g. when the capacity of an organizational unit is exceeded and a task must be transferred to another unit. Another related feature is the evaluation of functional integration also related with the completeness of the realised functions. The coordination effort between organizational units depends on the interdependence of the tasks they realise. Related tasks (aggregated in functions in the OBsim model) have a strong interdependence thus it is desirable that they are realised in the same organizational unit as to minimise the coordination effort. As in the OBsim model information flows with different interaction roles are treated accordingly, alternative scenarios of functional integration levels can be comparatively evaluated. During simulation the qualification structure can be dynamically modified. With this feature organizational unit learning can be simulated using a learning function dependent on time, organizational unit 
composition, frequency of task assignment change or another structural factor that the user can program.

\section{Boundary regulation}

Boundary regulation is the extent in which one organizational unit monitors and coordinates another. This is easily evaluated through the intensity of the coordination and monitoring information flows with organizational units interaction roles. As a result, self-regulation, one important factor for the organizational units autonomy, can also be assessed.

\section{Techno-organizational coupling}

Techno-organizational coupling is the extent to which the software system determines the activity of the organizational unit. A first evaluation of this criteria is made through the analysis of the model structures representing the task and software module that interact. A second level of evaluation is achieved during simulation through the monitoring of the amount of time that the task waits for an information output from the software module or waits to be allowed to input information into the software module. The techno-organizational coupling evaluation facility is of utmost importance in helping the specification and design of the software system.

\section{Technical linkage}

Following the approach described in function and task interdependence, it is possible to evaluate the interdependence between software systems both through the description of the information flows with software systems interaction roles and the intensity of that information flows at simulation time.

\subsection{Dificulties and limitations}

Information gathering for the model construction is the biggest difficulty in utilising this tool. For example, the average lead time of certain tasks like the production of a plan or the orders rescheduling because of a machine breakdown, cannot be easily quantified. The lack of accuracy in some tasks data is partially overcomed because the system is intended to comparatively evaluate scenarios and not to perform an absolute and accurate simulation of reality. Techno-organizational development doesn not rely exclusively on computer supported tools to analyse and specify the organization and production support software. Even in participating contexts, difficulties and hindrances in the systems development have to be surpassed taking care of social and power relations aspects. These aspects cannot be put in a computer model. Being conscious of this, OBsim can be a valuable analysis and colaboration instrument and function also as an "awareness tool" for those small budget projects in SME's where multidisciplinary development teams are not feasible.

\section{CONCLUSION AND FURTHER WORK}

Interdisciplinary techno-organizational development of IMS needs tools to shorten and improve the analysis and specification phases of the development life cycle. Moreover, trial and error methods are no longer feasible in organizational design, specially in SME's where time and resources are scarce. With the modelling and simulation system presented here, trial and error can be done with little costs for the manufacturing enterprise and a more comprehensive approach to the analysis and specification of IMS can be undertaken. In order to validate its underlying concepts, OBsim is now starting to be applied in supporting the introduction of PROFIT, a marketed advanced shop-floor management system developed 
under the ESPRIT project 5478 Shop-Control, in SME's. Further work includes the "plug-in" of a physical operations model as to enhance the simulated scenarios, and the incremental construction of an adequate class library based on several case studies. This work is part of more extensive research that is being undertaken in the development of modelling tools for techo-organizational optimisation of IMS at INESC/Porto.

\section{REFERENCES}

Bachman, R., Möll, G. (1994) Participating in CIM Systems, in Advances in Agile Manufacturing (ed. P.T. Kidd and W. Karwowski), IOS Press.

Bansler, J.P., Bodker, K. (1993) A Reappraisal of Structured Analysis: Design in an Organizational Context. ACM Transactions on Information Systems, 11, $\mathrm{n}^{\circ} 2$.

Emery, F.E. (1978) Characteristics of Socio-Technical Systems, in The Emergence of a new paradigm of work (ed. F. Emery), Australian National University, Canberra.

Ferreira, J.J., Mendonça, J.M. (1994) Supporting CIM Systems Life Cycle Through Material and Information Flow Executable Models in Proceedings of the 10th ISPE/IFAC International Conference on CAD/CAM, Robotics and Factories of the Future. Otawa.

Hoydalsvik, G., Sindre, G. (1993) On the Purpose of Object-Oriented Analysis, in OOPSLA'93 Proceedings, pp. 240-255.

Kensing, F., Munk-Madsen, A. (1993) Participatory Design: Structure in the Toolboox. Communications of the ACM, 36, No4, 78-83.

Paul, R.J, Thomas, P.J. (1994) Computer-Based Simulation Models for Problem-solving: Communicating Problem Understandings. Electronic Journal of Virtual Culture.

Soares, A.L., Mendonça, J.M. (1994) Interaction of Advanced Shop-Floor Management Systems with Production Systems Organization: An Exploratory Study, in Advances in Agile Manufacturing (ed. P.T. Kidd and W. Karwowski) - IOS Press.

Thompson, J.D. (1967) Organizations in Action. MacGraw-Hill, New York.

Weik, S., Grote, G., Zölch, M. (1994) KOMPASS, Complementary Analysis and Design of Production Tasks in Socio-Technical Systems, in Advances in Agile Manufacturing, (ed. P.T. Kidd \& W. Karwowski). IOS Press.

Zülch, G., Grobel, T. (1993) Simulating Alternative Organizational Structures of Production Systems, in Production Planning \& Control, Vol. 4, N ${ }^{\circ} 2,128-138$.

Williams, T.J. et al. (1993) Architectures for Integrating Manufacturing Activities and Enterprises, in Information Infrastructures Systems for Manufacturing (B-14). (Eds. H. Yoshikawa and J. Goossenaerts). Elsevier Science B.V. (North-Holland).

\section{BIOGRAPHY}

A. Lucas Soares was born in 1964 in Porto. He received the MsC. in Industrial Automation in 1991 and is currently assistant at the University of Porto. His interest areas are: technoorganizational development, human and social aspects of information technology and photography.

J.J. Pinto Ferreira was born in 1964 in Porto. He received the MsC. in Industrial Information Systems in 1991 and is currently assistant at the University of Porto. His interest areas are: enterprise integration and modelling, formal description techniques, distributed systems and windsurfing.

J. M. Mendonça was born in 1955 in Porto. He received the PhD in Electrical Engineering in 1985 and is currently Auxiliary Professor at the University of Porto. His interest areas are: manufacturing management, enterprise modelling, shop floor control and martial arts. 